\title{
A NOTE ON COMPLETE COLLECTIONWISE NORMALITY AND PARACOMPACTNESS
}

\author{
LOUIS F. McAULEY 1
}

1. A question that has aroused considerable interest and which has remained unanswered is the following. Is a normal Moore space metrizable? Both R. H. Bing and F. B. Jones have important results which go a long way toward answering this question. For example, Bing has proved that a collectionwise normal Moore space is metrizable [1] while Jones has shown that a separable normal Moore space is metrizable [2] provided the continuum hypothesis holds true. Although the results given in the present paper fail to answer the question at hand, it is shown that certain types of normality and paracompactness are equivalent in a Moore space, indeed, in more abstract spaces such as a semimetric topological space. ${ }^{2}$

2. Complete collectionwise normality. The reader is referred to R. H. Bing's paper [1] for definitions concerning screenability, collectionwise normality, discrete collections, and related concepts. We say that a collection $G$ is almost discrete if and only if it is discrete with respect to $G^{*}$ (the logical sum of the elements of $G$ ). Furthermore, a space is completely collectionwise normal if and only if for each almost discrete collection $G$ of point sets, there is a collection $H$ of disjoint open sets covering $G^{*}$ such that no element of $H$ intersects two elements of $G$.

A space is hereditarily collectionwise normal if and only if each subspace is collectionwise normal. It is not difficult to show that complete collectionwise normality is equivalent to hereditary collectionwise normality in a topological space.

A topological space $S$ is said to be $F_{o}$-screenable if and only if for each open covering $G$ of $S$, there exists a sequence $\left\{X_{i}\right\}$ such that (1) for each $i, X_{i}$ is a discrete collection of closed point sets each of which lies in an element of $G$ and (2) $\sum X_{i}$ covers $S$. There is an example [5] of a Hausdorff space satisfying the first axiom of count-

Presented to the Society, April 19, 1957; received by the editors February 10' 1958 and, in revised form, April 14, 1958.

${ }^{1}$ Preparation of this note was supported by NSF Contract G 4223 .

2 A topological space $T$ is said to be a semimetric topological space if and only if there is defined for $T$ a real valued non-negative distance function $d$ with the following properties: (1) $d(x, y)=d(y, x), x$ and $y$ in $T$, (2) $d(x, y)=0$ if and only if $x=y$, and (3) the topology of $T$ defined by spherical neighborhoods (which may not be open sets) is equivalent to the given topology of $T$, i.e., limit points are invariant. 
ability which is collectionwise normal but neither completely collectionwise normal nor $F_{0}$-screenable.

Using a proof analogous to one given by Bing for Theorem 9 of [1], the following lemma may be proved.

Lemma 1. A semimetric topological space $T$ is $F_{\sigma}$-screenable.

Proof. Let $\alpha$ denote a well ordering of an open covering $H$ of $T$. For each element $h$ of $H$, let $x(h, i)$ denote the set of all points $p$ such that (1) no element of $H$ which contains $p$ precedes $h$ in $\alpha$ and (2) there exists a $1 / i$-neighborhood of $p$ (spherical neighborhood, center $p$, radius $1 / i$ ) which lies in $h$. Now, let $X_{i}$ denote the collection of all such sets $x(h, i)$. It follows that $X_{i}$ is a discrete collection of sets. Furthermore, $\sum X_{i}$ covers $T$. It follows that $T$ is $F_{\sigma}$-screenable.

In the light of the appropriate definitions, a lemma due to Dowker [3], and the fact that strong screenability is equivalent to paracompactness in a regular topological space [7], it is not difficult to establish the following.

Lemma 2. A collectionwise normal $F_{\sigma}$-screenable topological space is strongly screenable and paracompact.

In a semimetric space, a closed set is a $G_{\delta}$ set. It then follows that a normal semimetric space is perfectly normal. Note that spherical neighborhoods in a semimetric topological space need not be open sets and that a semimetric may not be continuous.

Using a number of known results, we are able to establish equivalence between complete collectionwise normality and paracompactness in a semimetric space as stated below.

Theorem 1. Suppose that $S$ is a semimetric topological space. Then a necessary and sufficient condition that $S$ be paracompact is that $S$ be completely collectionwise normal. Indeed, the following implications hold true in $S:$ hereditary collectionwise normal $\rightarrow$ collectionwise normal $\rightarrow$ paracompact $\rightarrow$ hereditary paracompact $\rightarrow$ hereditary collectionwise normal.

Proof. The sufficiency follows by use of the preceding lemmas.

Suppose that $S$ is paracompact. Then $S$ is normal and perfectly normal. Now, a result [2] due to C. H. Dowker yields that $S$ is hereditarily paracompact. R. H. Bing has shown that a paracompact topological space is collectionwise normal [1, Theorem 12]. Hence, $S$ is completely collectionwise normal. The truth of Theorem 1 is evident.

A developable space [1] is a semimetric topological space and each 
Moore space [1] is a regular developable space. Thus, from Theorem 1 , it follows that complete collectionwise normality is equivalent to paracompactness in such spaces.

3. Pointwise paracompactness, separability, and complete collectionwise normality. It seems to remain unknown whether a normal semimetric topological space $S$ is paracompact. If such a space is paracompact, then a normal Moore space is metrizable. Jones has shown [4] that a normal separable Moore space is hereditarily separable and metrizable provided the continuum hypothesis holds true. In Theorem 3, we replace this condition by pointwise paracompactness. Theorem 2 indicates further the similarity of semimetric spaces to metrizable Moore spaces though not being always metrizable.

Theorem 2. A normal, pointwise paracompact, and separable semimetric topological space $S$ is hereditarily separable, completely collectionwise normal, and paracompact.

The space $S$ may fail to have a countable basis [4, Example 3.1].

Proof of Theorem 2. Suppose that $G$ is an open covering of $S$. Since $S$ is pointwise paracompact, there exists an open refinement $H$ of $G$ such that the star of each point $p$ with respect to $H$ is finite, i.e., the collection of all elements of $G$ which contain $p$ is finite.

Let $N=\sum p_{i}$ be a dense countable subset of $S$. Since the star of $p_{i}$, for each $i$, w.r.t. $H$ is finite, it follows that there is a countable open refinement $K$ of $G$. Hence, $S$ has the Lindelöf Property. It follows that $S$ is hereditarily separable. A theorem [8] due to Morita implies that $S$ is paracompact and hence completely collectionwise normal.

Theorem 3. A normal, separable, and pointwise paracompact Moore space $S$ is metrizable.

Proof. By Theorem 2, $S$ is paracompact. Now, $S$ is collectionwise normal by use of Theorem 1 and metrizable by a theorem [1] due to Bing.

\section{REFERENCES}

1. R. H. Bing, Metrization of topological spaces, Canad. J. Math. vol. 3 (1951) pp. $175-186$.

2. C. H. Dowker, An imbedding theorem for paracompact metric spaces, Duke Math. J. vol. 14 (1947) pp. 639-645.

3. - On a theorem of Hanner, Ark. Mat. vol. 2 (1952) pp. 307-313.

4. F. B. Jones, Concerning normal and completely normal spaces, Bull. Amer. Math. Soc. vol. 43 (1937) pp. 671-677.

5. L. F. McAuley, Paracompactness and an example due to F. B. Jones, Proc. Amer. Math. Soc. vol. 7 (1956) pp. 1155-1156. 
6. - $A$ relation between perfect separability, completeness, and normality in semimetric spaces, Pacific J. Math. vol. 6 (1956) pp. 315-326.

7. E. A. Michael, $A$ note on paracompact spaces, Proc. Amer. Math. Soc. vol. 4 (1953) pp. 831-838.

8. - Another note on paracompact spaces, Proc. Amer. Math. Soc. vol. 8 (1957) pp. 822-828.

9. K. Morita, Star-finite coverings and the star-finite property, Math. Japon. vol. 1 (1948) pp. 60-68.

UNIVERSITY OF WISCONSIN 